\title{
To the Lighthouse: Embracing a Grand Challenge for Cancer Education in the Digital Age
}

\author{
David Wiljer ${ }^{1,2}$ \\ Published online: 8 May 2020 \\ (C) American Association for Cancer Education 2020
}

Over the past several months, with the emergence of one of the largest and most devastating pandemics the world has ever experienced, COVID-19, health care has rapidly been transformed around the world. One of the most significant transformations has been to a wide scale adoption of virtual and digital care, work and learning. As we make the shift, we are adopting new ways of caring and learning, but we must not lose sight of fundamental values, like providing compassionate, safe, and high-quality care and education.

In a recent web television series featuring a rather eccentric music conductor, Mozart in the Jungle, the conductor, Rodrigo, is asked to perform Mozart's unfinished Requiem. Rodrigo is full of angst and anxiety about the performance. The requested version of the piece was completed by a very special composer and conductor, WAM (Wolfgang Amadeus Mozart), an artificial intelligence expert system that has been fed the data of every Mozart composition and recording. WAM also conducts a piece, which is done too perfectly and then an incensed Rodrigo throws WAM into a nearby stream (WAM is a computer program and so virtually unharmed). Rodrigo then conducts the Requiem but strips the piece bare of its AI perfections. While performed beautifully, the donor is disappointed that Rodrigo set humanity back in its collective pursuit of progress.

How many clinicians and cancer educators can empathize with Rodrigo? The world is changing so fast around them that they are no longer practicing health care in the same way that they were trained and perhaps are now extremely uncomfortable in their new environment. Atul Gawande described the undelivered promises of the digitization of health care in the now famous piece Why Doctors Hate Their Computers, in which he states, "a system that promised to increase my mastery over my work has, instead, increased my work's mastery

David Wiljer

David.Wiljer@uhn.ca

1 Department of Psychiatry, Institute of Health Policy Management and Evaluation, University of Toronto, Toronto, ON, Canada

2 UHN Digitial, University Health Network, Toronto, ON, Canada over me." [1]. In the evolution of the digitization of health care, the emergence of AI in many facets of health and cancer care is raising questions about how we will work with $\mathrm{AI}$ to provide the best quality care to our patients. This is certainly a critical issue of our time. Will we embrace this new technology or cast it aside as Rodrigo did and seek the safety of the known, familiar and sacred?

Eric Topol has demonstrated that AI has the potential to change almost every aspect of health care. He has argued that applications are quickly evolving in the entire continuum of care, from embryo selection and early detection of genetic disorders to preventive, diagnostic, and predictive care [2]. Several studies have recently demonstrated that AI systems are as good or equal to human experts in areas such as in the reading of imaging studies and in the diagnosis of certain skin lesions [3, 4]. But, early in 2020, an important and seminal paper was published in Nature demonstrating that in the diagnosis of breast cancer, the AI system outperformed the human experts in several respects [5]. This study raises some very important questions about the relationship between $\mathrm{AI}$ and experts and how care should be delivered. If these systems are outperforming traditional diagnostic methods, do we not owe it to our patients to utilize the best tools available? In addition, it is important to note that in Topol's scan of the AI literature, almost half the studies were related to the cancer field [2]. Cancer clinicians and educators will be on the front line of the advent of AI in health care and therefore need to move quickly in order to be prepared. We then need to ask ourselves, what role should we be prepared to play as cancer educators in the integration of AI into cancer care?

The pathway to the appropriate integration of $\mathrm{AI}$ into our organizations and the delivery of care is, at this point, unclear. Fountaine et al. have written a playbook for organizations to guide the adoption of AI. While they present clear options for success in multiple contexts, there is one simple common denominator for all organizations: there is a need to educate everyone - a certain level of AI literacy is essential at all levels of the organization [6]. In a recent article, we have argued that the creation of an AI-enabled health care 
organization requires building awareness and capacity, gaining experience in developing and/or implementing $\mathrm{AI}$ initiatives and innovations, establishing strategic partnerships, and mobilizing and exchanging rapidly emerging knowledge and science related to AI [7]. In addition, a certain level of AI literacy is required across the organization. The knowledge domains include (1) data governance and ethical principles, (2) basic statistics and the science of algorithms, (3) data visualization and data information exchange, and finally (4) an understanding of how AI will transform the processes related to care [7]. Cancer educators must not only develop these skills but also take a leading role in ensuring that organizations are prepared to implement AI technologies in an appropriate and responsible manner.

There is an enormous risk that as we relentlessly pursue progress we can leave the most vulnerable behind and lose sight of the importance of compassionate care for our cancer patients. The National Academy of Medicine (NAM) has just released a seminal document entitled, AI in Healthcare: The hope, the hype, the promise and the peril [8]. This document argues for "significant training or retraining of the workforce (Matheny, p. 104)" and the required shifts in entry to practice programs as well as professional development in the relevant science, engineering, and math literacy. The authors advocate for using the new model Quintuple Aim of Equity and Inclusion, focusing on AI, to reduce cost, improve population health, increase care team well-being, as well as enhancing the patient experience through equity and inclusion. Given the complexity of AI systems and the potential for reinforcing known bias, health care professionals will need the required education to take an active role in understanding and working with these new emerging technologies for the benefit of all patients. I would argue, however, that it is beyond the notion of equity and inclusion. In the evolution of $\mathrm{AI}$ in cancer care, we should challenge ourselves to use the technologies to provide compassionate, high quality care. The notion of digital compassion and digital empathy should evolve. We must educate health care professionals to have the capabilities to harness these tools to provide compassionate care and, at the same time, we should build the tools with the end-goal of compassionate care in mind $[9,10]$.

The emergence of new digital tools, the growth of data, and the emergence of artificial intelligence represent great opportunity for transformational change, and yet these technologies threaten the very existence of traditional healthcare as we know it. Can we imagine a day when our health care is delivered together with machines [11]? The great Virginia Woolf wrote an amazingly poetic piece about a journey to an elusive lighthouse which serves as a literary exploration of the human condition in the face of uncertainly and rapid change. One of the characters, Lily Briscoe, attempts to document the journey to the lighthouse in a painting and the narrators tells us that after great effort, the lighthouse "had become almost invisible". Lily has a final moment of despair in her journey and then "...she looked at her canvas; it was blurred. With a sudden intensity, as if she saw it clear for a second, she drew a line there, in the centre. It was done; it was finished. Yes, she thought, laying down her brush in extreme fatigue, I have had my vision" [12]. The use of AI in health care can be equated to the journey to the lighthouse. On some days, the journey is too overwhelming to contemplate, but with a quest for understanding, we may have some precious moments of clarity.

For cancer educators, the advent of AI represents a grand challenge. The time has come for us to think about how the opportunity for augmented intelligence of cancer providers will transform the care our patients receive. The journey to the AI lighthouse will not be clear, it will not be easy, we will experience fatigue in braking through the haze to achieve a clear vision. But the literal or figurative dismemberment of AI in healthcare would come at the cost of progress and better patient care. And, thus, we must prepare our current and future providers to operate safely and compassionately in the new world of AI and augmented intelligence.

\section{References}

1. Gawande A (2018) Why doctors hate their computers. The New Yorker, $\mathrm{p} 12$

2. Topol EJ (2019) High-performance medicine: the convergence of human and artificial intelligence. Nat Med 25(1):44-56

3. Esteva A, Kuprel B, Novoa R, Ko J, Swetter S, Blau HM, Thrun S (2016) Dermatologist-level classification of skin cancer. Nature

4. Rabbani M, Kanevsky J, Kafi K, Chandelier F, Giles FJ (2018) Role of artificial intelligence in the care of patients with nonsmall cell lung cancer. Eur J Clin Investig 48(4):e12901

5. McKinney SM, Sieniek M, Godbole V, Godwin J, Antropova N, Ashrafian H, Back T, Chesus M, Corrado GC, Darzi A (2020) International evaluation of an AI system for breast cancer screening. Nature 577(7788):89-94

6. Fountaine T, McCarthy B, Saleh T (2019) Building the AI-powered organization. Harv Bus Rev 63-73

7. Wiljer D, Hakim Z (2019) Developing an artificial intelligenceenabled health care practice: rewiring health care professions for better care. J Med Imaging Radiat Sci 50(4):S8-S14

8. Matheny M, Israni ST, Ahmed M, Whicher D (2020) Artificial intelligence in health care: the hope, the hype, the promise, the peril. Natl Acad Med 94-97

9. Kemp J, Zhang T, Inglis F, Wiljer D, Sockalingam S, Crawford A, Lo B, Charow R, Munnery M, Takhar SS (2020) Delivery of compassionate mental health care in a digital technology-driven age: scoping review. J Med Internet Res 22(3):e16263

10. Wiljer D, Charow R, Costin H, Sequeira L, Anderson M, Strudwick G, Tripp T, Crawford A (2019) Defining compassion in the digital health age: protocol for a scoping review. BMJ Open 9(2):e026338

11. Meskó B (2019) The impact of digital health technologies on the future of medical specialties in one infographic. Med Futurist [https://medicalfuturist.com/towards-creativity-in-healthcare-theimpact-of-digital-technologies-on-medical-specialties-in-aninfographic?utm source $=$ The $\% 20$ Medical $\% 20$ Futurist $\%$ 20 Newsletter\&utm_campaign $=$ b0 07238 ce29-EMAIL_ 
CAMPAIGN_2019_08_12\&utm_medium $=$ email\&utm_term $=0$ efd6a3cd08-b07238ce29-420645589. Accessed September 1st

12. Woolf V (1981) To the lighthouse. 1927. San Diego: HarvestHarcourt
Publisher's Note Springer Nature remains neutral with regard to jurisdictional claims in published maps and institutional affiliations. 